(703 Tob. Mayer) beobachtet; der Tag der Beobachtung war aber nicht, wie es dort heifst, der 19., sondern der 20. Oct. Die Rectase. des Sterns nahm ich aus der Hist. cél. S.171, für $1800=266^{\circ} 2^{\prime} 19^{\prime \prime}, 3$, Decl. $-18^{\circ} 45^{\prime} 6^{\prime \prime}, 9$.

Bedeckung 1792. 20. Oct. Eintritt am dunkeln Mondrande.

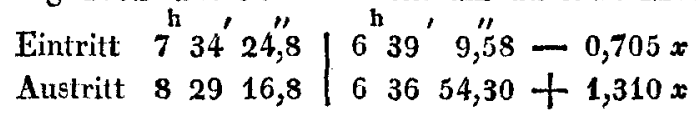

Der Eintritt wird als sehr zuverlässig angemerkt; der Austritt am hellen Mondrande scheint es desto weniger gewesen zu seyn, und da der Austrilt, was nicht zu erwarten war die Conjunction sogar um einige Minuten früher gibt, als der Eintritt, so ist beinahe zu vermulhen, dafs ein Druck- oder Schreibfehler Statt gefunden haben möchte, und dafs es statt: $8^{\text {h }} 44^{\prime} 32^{\prime \prime}$ wahre Zeit des Austritts heifsen sollte: $8^{\mathrm{b}} 46^{\prime} 32^{\prime \prime}$. Für Paris ist die berechnete Conj. in mittl. Zeit $=19$ Oct.

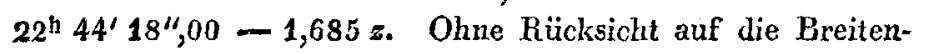
correction $x$, welche sich durch den Ein- und Austritt $+67 ", 14$, alsu viel zu grofs ergeben würde, findet man demnach die Länge des Beobachtungsortes +7 h $54^{\prime} 51^{\prime \prime}, 58$ $-0,705 x+1^{\prime \prime}, 685 z$ durch den Eintr. am dunkeln Mondrande, und, auf die Cathedrale zu Manila reducirt, $+7^{\text {h }} 54^{\prime} 36^{\prime \prime}, 88$. Le Gentil fand frïher aus Verfinsterungen der Jupiterstrabanten, von Lalande durch gleichzeitige Beobachtungen ver-

Stuttgart, 7 Mai 1828. bessert, die Länge von Manila $+7^{\text {h }} 54^{\prime} 4^{\prime \prime}, 5$ (Mon. Corr. IIIB. S.170). Dagelet setzt die Lünge von Cavita, dem Hafen von Manila $+7^{\text {h }} 50^{\prime} 0^{\prime \prime}$ und La Pérouse, von dieser Angabe sehr abweichend, $+7^{\text {h }} 55^{\prime} 22^{\prime \prime}, 7$ (s. oben bei Buenos Ayres). Herr Prof. Oltmanns, der obige Sternbedeckung mitgetheilt hat, findet (Mon. Corresp. XXVII B. S. 324) aus mehreren von Malaspina und Don Juan Vernaci beobachteten Ein - und Austritten der Jupiterstrabanten, durch die Berechnung nach den Tafeln ohne correspondirende Beobachtungen, die Länge $127^{\circ} 15^{\prime}$ östlich ron Cadix, oder, meine Bestimmungen der Länge von Cadix rorausgesetzt, $+7^{\text {h }} 54^{\prime} 29^{\prime \prime}$ in Zeit von Paris. Es gelang mir indefs, za dem Austritte des ersten Trabanten, am 6 März 1792 von IMalaspina beobachtet, eine correspondirende Beobachtung von Hrn. Flaugergues in Viviers aufzufinden; sie steht im I Supplementbande zu den Berl. astron. Jahrb. S.128, und gibt unmiltelhar die Länge von Manila $+7^{\text {h }} 54^{\prime} 21^{\prime \prime}$. Durch den Venusdurchgang von 1769 findet endlich Herr Prof. Encks (s. dessen Abhandlung über diesen Durchgang S.112) aus der Beobachtung der innern Berührung beim Austritt in Manila die Länge $+7^{\text {h }} 54^{\prime} 23^{\prime \prime}, 6$. Mit den drei letzteren Bestimmungen, so wie mit der Connaissance des tems; welche $+7^{\mathrm{h}} 54^{\prime} 32^{\prime \prime}$ gibt, stimmt demnach das Resultat der oben berechneten Sternbedeckung ziemalich nahe überein.

\title{
Ueber die Länge von Manila, Port Bowen, Funchal, Cap Frio und Rio Janeiro.
}

\section{M a n i 1 a.}

$I_{m}$ vorhergehenden Aufsatze habe ich die Länge Manila's aus einer von Malaspina am 20 Oct. 1792 beobachteten Stepnbedeckung berechnet. Sehr angenehm wurde ich vor einigen Tagen durch eine Berechnung derselben Bedeckung von Herrn Daussy in der Conn. des tems pour 1830 p. 41 iiberrascht. Die Resultate stimmen genau überein; Herr Daussy findet aus dem Eintritte die Länge der Cathedrale in Manila $7^{\text {h }} 54^{\prime} 36^{\prime \prime}, 8+1,684 z$, ich fand letzhin $7^{\text {h }} 54^{\prime} 36^{\prime \prime}, 88$ $+1,685$ z. Malaspina hat indefs, wie ich aus der Conn. des tems am angef. Ort sehe, am 5. März 1792 noch eine andere Sternbedeckung in Palapay (Insel Samar) beobachtet, wodurch mittelst des bekannten Längenunterschicdes gleichfalls die Länge von Manila bestimmt werden kann. Ich habe nun auch noch diese Bedeckung berechnet, und mich dabei absichtlich fuir den Ort des Mondes und des Sterns derselben Elennente, wie Herr Daussy, bedient, nur dafs ich, wie gewöhnlich, die Erdabplattung, welche dieser $=\frac{1}{3} \frac{1}{\delta}$ setzt, $2 u \frac{\pi}{3} \frac{1}{10}$ angenummen habe; Länge und Breite des Monds sind durch Greenwicher Beobachtungen vom 4, 5, 7, 8 März verbessert.

Palapay (Breite $+12^{\circ} 37^{\prime} 7^{\prime \prime}$ ). Bedeckung von o Löwe; 414 T. Mayer, am 5 März 1792. Eintritt am dunkeln Mondrande.

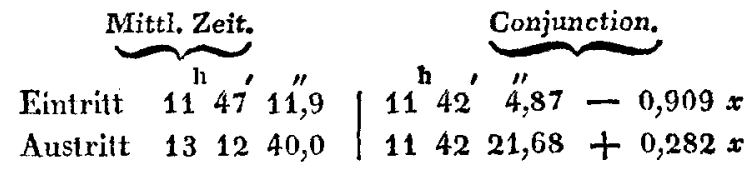

Durch die Conjunctionen des Ein - und Austritts findet sich die Correction $x=-14^{\prime \prime}, 114$, oder, da man wohl berech. tigt seyn dürfte, von der Zeit des Austritts am erleuchteten Mondrande 6 Secunden abzuziehen, $x=-9^{\prime \prime}, 076$. Die Berechnung gab: Conj. in Paris $=3^{\text {h }} 31^{\prime} 17^{\prime \prime} ; 24-1,904$, und so erlält man aus der verbesserten Conj. in Palapay $11^{\mathrm{h}} 42^{\prime} 13^{\prime \prime}, 12$, die Länge von Palapay $8^{\mathrm{h}} 10^{\prime} 55^{\prime \prime}, 88+1,904 z$ und daraus, da die Cathedrale zu Manila 16.11"4, in Zeit westlicher liegt, die Länge von Manila $7^{\text {h }} 54^{\prime} 44^{\prime \prime}, 48+1,904$ zo 
Hexr Datssy fand die Conj. aus dem Eintr. $11^{\text {th }} 42^{\prime} \mathrm{I}^{\prime \prime}, 2$ $+0,91 x$, aus dem Austr. $11^{\text {h }} 42^{\prime} 24^{\prime \prime}, 6-2,9 x$. und leitet daraus, durch den blofsen Eintritt, aber ohne die Conjunction durch den Werth von $x$ zu verbessern, die Lünge voll Manila $=7^{\mathrm{h}} 54^{\prime} 32^{\prime \prime}, 4 \mathrm{ab}$; die Conjunction für l'aris hatte er $=7^{\mathrm{h}} 31^{\prime} 17^{\prime \prime}, 2$ berechnet. Da aber auch die Breite des bedeckten Sterns einiger Verbesserung, dic in der Correction $\boldsymbol{x}$ mit eingeschlossen ist, bediurfen, und da selbst die Mondsbreite, obgleich durch Beobachtungen verbessert, noch kleine Berichtigungen nöthig haben könnte, zumal da die Greenwicher Beobachlungen nur eine zwischen - 3",5 und + 4",6 fallende Verbesserung derselben andeuten; so schien es mir sicherer, die Correction $x$ nicht ganz zu vernachlïssigen. Der Werth von $x$, aus Hrn. Datussy's Berechnungen abgeleitet, würde, wenn man, wie oben, rom Austritte 6 Sec. abzieht, $=+14^{\prime \prime}, 50$ gefunden werden, und damit die verbesserte Conjunction des tintritts $=11^{\text {h }} 42^{\prime} 14^{\prime \prime}, 40$ : daraus folgt die Tü̈nge von. Manila $7^{\text {h }} 54^{\prime} 45^{\prime \prime}, 80$ nur $1^{\prime \prime}, 32$ gröfser, als nach meiner Berechnung. Durch sorgfältige Discussion melurerer in Manila beobachteten Ein - und Austritte der Jupiterstrabanten bestimmt Hi. Daussy liese Länge zu $7^{\text {h }} 54^{\prime} 45^{\prime \prime}$, hält sich aber am Ende blos an die zwei von ihm berechneten Eintritle der Sternbedeckmingen, welche $7^{\text {h }} 54^{\prime} 36^{\prime \prime}, 8$ und 7 in $54^{\prime} 32^{\prime \prime}, 4$ gaben, in Mittel also 7 h $54^{\prime} 34^{\prime \prime}, 6$. Da die Stembudeckung vom 20 Oct. 1792 unmiltelbar die Länge von Manila gibt, die rom 5. Mïrz blos durch Uebertragung der Zeit von Palapay; so gebe ich dem fiesultate aus der letztern Bedeckung nu den dritien Theil des Werths der erstern, und erhalte damit für die Länge der Cathedrale von Manila den genäherten Werth $=7^{\text {b }} 54^{4} 38^{\prime \prime}, 8$.

\section{Port Bowen.}

Die Kenutnifs einer an diesem Orte beubachteten Sternbedeckung verdanke ich Herrn $\nu$. Heiligenstein in Mannhein, der mir solche ans den Denkschrifien der Londoner astronomischen Societä milgetheilt hat. Port Bowen ist der Ort, wo sich die Schiffe einer Nordpolexpedition nem Mo. nathe lang aufinelten, ohne Zweilel der nördlichste Punct, auf dem jemals Sternbedeckingen beobachtet wurden; die Breite ist, obigen Schriften zufolge, $73^{\circ} 13^{\prime} 39^{\prime \prime}, 4$, die Länge - $5^{\text {h }} 55^{\prime} 39^{\prime \prime}, 2$ von Greenwich, demnach $-6^{\text {h }} 5^{\prime} 0^{\prime \prime}, 8$ in Zeit von Paris. Ob diese Bedeckming schon irgendwo berechnet worden, ist mir nicht bekannt.

Bedeckung von Fi. 3 im Krebs: 1825. 1 Tebr. Eintritt am dunkeln Mondrande.

$$
\text { Einlrilt } 11^{\mathrm{h}} 44^{\prime} 58,26 \mid 12^{\mathrm{h}} 10^{\prime} 34,67-0,479 x
$$

Fur Paris ist dit berechnete Conjunction $=18^{\text {h }} 25^{\prime} 31^{\prime \prime}, 80$ - 1,636z, daher Länge von Port Bowen - 6h $4^{\prime} 57^{\prime \prime}, 13$
$-0,479 x+1,636 z$, nur wenig von obiger Angabe der lünge verschieden.

Funchal auf Mladeira.

Auf der Reise nach Neusüdwales hat Herr Prof. Rü̈ker cine Sternbedeckung auf dem Thurme zu Funchal beolsachtet, die ich, so wie die übrigen auf dieser Scereise beohachteten Bedeckungen, welche unten folgen, in Rechnung genommen habe. (Astr. Nachr. Nr. 5.)

Bedeckung von $\psi$ Junglrau 1821. 9 Jun. Eintritt am dunkeln Mondrande.

$$
\text { Eintritt } 9^{\mathrm{h}} 38^{\prime} 8,17 \mid 9^{\prime \prime} 41^{\prime} 42^{\prime \prime}, 92+2,196 x
$$

Für Paris berechnete ich die Conjunction $=10^{\mathrm{h}} 5 \mathrm{~S}^{\prime} 45^{\prime \prime}, 58$ $-2,031 z$; diefs gibt die Länge von Funchal $=-1^{\mathrm{h}} 17^{\prime} 2^{\prime \prime}, 66$ $+2,196 x+2,031 z$. Nur, wenn $x$ einen geringen Werth hälte, könnte dieser Längenbestimmung einige Zuverlässigkeit zugeschrieben werden. Horr Rümker sagt, olme nähere Umstände der Berechnung anzugeben, dafs er aus dieser Bedeckung die Länge $-1^{\text {h }} 7^{\prime} 36^{\prime \prime}$ von Greenwich gefunden habe, demnach $-1^{\text {b }} 16^{\prime} 57^{\prime \prime}, 6$ von Paris, chronometrische Bestimmungen gaben ilm $-1^{\text {h }} 17^{\prime} 0^{\prime \prime}, 2$ (nahe, wie oben die Sterubedeckung) und neun Mondsdistanzen - $1^{\text {h }} 16^{\prime} 38^{\prime \prime}, 6$. In der Conn. des tems pour $1830^{\circ}$ p. 249 hat Herr Capitain Duperrey eilf verschiedene, meist auf Seereisen gemachte Beslimmungen fïr die Länge von Funchal zusammengestellt; das Mittel daraus ist $-1^{\text {h }} 16^{\prime} 58^{\prime \prime}, 2$ und die neueste diesen" Bestimunungen durci Dr. Tiarka, welcher nach Auftrag der Board of Longitude in J. 1823 mit 16 Chronometern diese: Länge untersucht hat, gibt $-1^{h} 17^{\prime} 0^{\prime \prime}, 3$, was dem obigen Resullate der Bedeckung (vielleicht zufäligerweise) selur nahe tommt.

\section{Cap Frio (Küste von Brasilien).}

Eine von dem ehemaligen Gouverneur, Thomas Brisbane, im Angesichte vom Cap Frio und bei einer ziemlich schnellen Bewegung des Schiffes, auf der See beobachtete Sternbedeckung, die nicht länger als 10 Minuten dauerte, schien mir wenigstens eine genanere Berechnung zu verdienen, wenn über ihron eigentlichen Werth entschieden wer. den sollte. (s. Asti. Nachr. Nr. 5.)

Bedeckung des Regnius 1821. $30 \mathrm{Jul}$. Eintritt am dunkeln Mondrande.

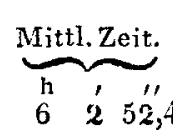
Ort des Schiffes

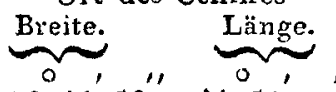

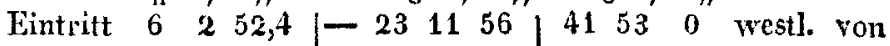

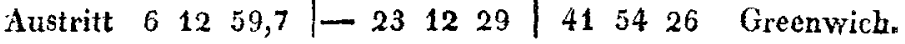
Daraus berechnete ich unmittelbar für den Ort des Schiffes: Conj. durch den Eintr. 41810,08 + 24,086x-24,162 da - Austr. $4185,74+4,450 x-4,845 d a$ 
Nachdem ich den Austritt auf den Ort des Schiffes beim Eintritte, demnach Ein- und Austritt auf einerlei geographischer Punct, reducirt hatte, so erhielt ich, mit dem reducirten Austritte $6^{\text {h }} 12^{\prime} 56^{\prime \prime}, 9$, die Conjunction durch den Austritt $4^{\text {h }} 18^{\prime} 3^{\prime \prime}, 47+4,453 x$. Nun geben Ein - und Austritt die Correction $x=-0$ ",3367 und damit die blos durch $x$ verbesserte Conjunction für den Ort des Schiffes beirn Eintr. $4^{\text {li }} 18^{\prime} 1^{\prime \prime}, 97$, oder, da ,mach chronometrischen Bcobachtungen das Cap Frio 16 " in Zeit westlich von der Lünge des Schiffs beim Eintrittt entfernt war, Conjunction fuir das Cap Frio $4^{\text {h }} 17^{\prime} 45^{\prime \prime}, 97$. Fïr Paris berechnete ich die Conjunction $=7^{\text {h }} 14^{\prime} 11^{\prime \prime}, 41-1,909 z$ : daraus folgt die Länge des Cap Frio $=-2^{\mathrm{h}} 56^{\prime} 25^{\prime \prime}, 44+24,162 d x+1,909 z$ durch den Eintritt. Da sich voraus vermuthen liefs, dafs auch der Coëficient der Verbesserung des Mondhalbmessers a eine beträchtliche Gröfse haben werde, so unterliefs ich nicht, denselben gleichfalls zu berechnen. Nach der Conn. des tems pour 1825 p. 335 hat Brisbane selbst diese Bedeckung berechnet, und aus derselben die Länge des Cap Frio $=-2^{\mathrm{h}} 57^{\prime} 15^{\prime \prime}, 33$ gefunden. Gegen eine Längenbestimmung aus dieser Bedekkung lassen sich allerdings sehr erhebliche Einwendungen machen. Vors erste mufs der Austritt ganz verworfen werden, da, wie der Beobachter anmerkt, Regulus nach dem Austritte, der ïberdiefs ann erlcuchteten Mondrand Statt hatte, nicht blos auf Secunden, sondern 2 Minuten lang weit hinein auf der Mondscheibe sichtbar war. Der Eintritt am dunkeln Mondrande mag genauer beobachtet seyn; dafs aber auch dieser, die Länge zu bestimmen, keincswess tauglich ist, lehrt schon ein Blick auf dic sehr starken Factoren der beiden Correctionen der Breite und des Halbmessers des Monds; die fïi $x$ gefundene Correction wird, insofern der Austritt Einflufs auf dieselbe hat, etwas ungewifs, und der Werth von $d x$ bleibt völlig unbekannt, ebenso wie der Werth yon z. Weniger verwerflich scheint mir dicse Bedeckung aus dem Grunde, dafs, wie in der Conn. des tems 1830. p. 147 behauptet wird, die Entfernung des Schiffes vom Cap Frio zu wenig genau bestimmt gewesen sei, um das Resultat der Beobachtung mit Sicherheit auf das Cap beziehen zu können. Die Sonne gieng damals für die Brcite des Schiffs, wie ich durch Rechnung fand, um $5^{\text {ht }} 33^{\prime}$ mittl. Zeit unter, und der Eintritt fiel nur eine halbe Stunde später, so dafs das Cap noch gut sichtbar seyn mufste. Auch veründerte sich während der 10 Minuten zwischen dem Ein - und Austritt die Länge des Schiffes nur um 1' $26^{\prime \prime}$ im Bogen, und da das Schiff 8,6 Meilen *) in einer Stunde segclte, so konnte bei der Lage des Cap gen

*) In der Conn. d. tems 1830, p.14S Anmerk. wird vermuthet, dafs statt 8,6 Lieves stehen sollte: 8,6 Milles: allein ohne Zweifel sprach der Brief des Hrn. Ritter olbers eben so, wie A. N. Nr.5 von Meilen, die erst der Uebersetzer in Lieves verwandelte.
Norden, eine Meile Fehler in der geschätzten Entfermung nicht mebr als eine lalbe Raumminute Fohler in der Länge zur Folge haben. Doch ich habe hereits selbst die ganze Bedekkung für unbrauchbar erklärt, und beniitze gerne die Elemente, wclche Herr Givry, der obigen Einwurf in der Conn. d. tems gemacht hat, zu einer zuverläss geren Bestinmung der Länge des Cap Frio eben daselbst mittheilt. Nach Hrn. Givry liegt das Cap östlich von Pain du Sucre (dem Zuckerhuthlï̈gel)

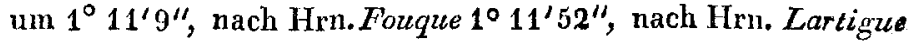
$1^{\circ} 10^{\prime} 39^{\prime \prime}$, im Miltel also $1^{\circ} 11^{\prime} 13^{\prime \prime}, 3$ von Pain du Sucre, oder um $1^{\circ} 11^{\prime} 46^{\prime \prime}, 3$ östlich von der Insel Ratos (Station von Rio Janeiro). Die Länge der Insel Ratos (s. unten die Berechmingen für Rio Janeiro) ist im Mittel aus älferen und neueren Bestimmungen $-3^{\text {h }} 2^{\prime} 15^{\prime \prime}$, demnach Länge des Cap Frio $=-2^{h} 57^{\prime} 28^{\prime \prime}$. Die Bedeckung des Regulus, nach meiner Berechnung gab also das Cap um eine ganze Minute in Zeit zu östlich; näher stimmt das Resultat von neun durch die Herren Rümker und Brisbane gemessenen Monddistanzen, welche $-2^{\text {b }} 57^{\prime} 9^{\prime \prime}, 6$ gaben.

\section{Rio Janeiro.}

Becleckung der zwei Sterne eines Doppelsterns 1821. 6 Aug. Eintritt am dunkeln Mondrande.

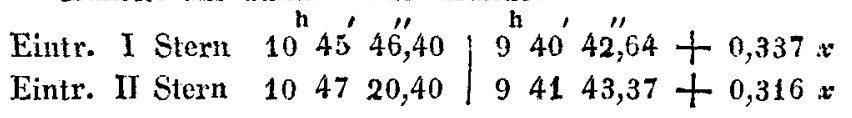

Den einen dieser Sterne, welche den Doppelstern bilden, und deren Eintritt von Sir Th. Brisbane beobachtet wurde, hat Hr. litler Dr. Olbers in der Hist. cél. p. 472 richtig nachgewiesen; ich fand dessen AR. für $1800=230^{\circ} 20^{\prime} 21^{\prime \prime}, 1$ und dic Decl. $-23^{\circ} 47^{\prime} 57^{\prime \prime}, 7$. Da der andere Stern in der Hist. cél. fehlt, so berechnete ich oben die Conj. in der gedoppelten Hypothese, dafs entweder der erste oder der zweite eingetretene Stern mit dem in der Hist. cél. vorkommenden identisch seyn müsse, und bestimmte für den letzteren die Conj. in Paris $=12^{\text {h }} 44^{\prime} 54^{\prime \prime}, 55-1,984 z$. Die ersle Hypothese gab die Länge von Rio Janeiro (Botofogo) $-3^{\text {h }} 4^{\prime \prime 1} 1^{\prime \prime}, 91$ $+0,337 x+1,984 z$., und die zweite Hypoth. $-3^{\text {h }} 3^{\prime 1} 11^{\prime \prime}, 18$ $+0,316 x+1,984 z$. Da bei der ersten Voraussetzung die damit zusammenhängende Länge gar zu sehr von der nahe bekannten abweichen würde, so ist es sehr wahrscheinlich der zweite, oder der später eingetretene Stern, der dem Sterne der Hist. cél. entspricht. Aber auch in dieser Hypothese erscheint die Länge noch etwa un 1 Min. Zeit zu westlich. Der Beobachter sellbst war wegen trüber Witterung etwas zweifelhaft; ein Stern der 7.8 Gröfse konnte bei getrïbtem Himmel wohl viele Secunden früher, als or wirklich eintrat, scheinbar verschwunden seyn, und dieser Umstand zur wahrscheinlichen Erklärung einer allzu westlichen Länge dienen. 
Für Orte, wo nur selten beobachtet wird, sind auch solche Beobachtungen, anf welche sonst weniger Werlh gelegt wird, nicht unwichtig; ich berechnete daher auch die nachfolgende altere Sonnenfinsternils, obschon nur der Anfang derselben, und zwar, wie der Beobachter, der portugiesische Astronom Dorta sclbst bemerkt, ganz nahe am Horizonte wahrgenommen werden kounte.

Sonnenfinsternifs 1784. $20 \mathrm{Febr}$. in Rio Janciro beobachtet. (Mon. Corr. IV B. S. 354).

$\Lambda$ fang $6^{\text {h }} 5^{\prime} 20^{\prime \prime}, 4 \mid 5^{\text {h }} 45^{\prime} 37^{\prime \prime}, 33-0,007 x$.

Für Paris ist die berechnete Conjunction: $8^{\text {l }} 47^{\prime} 47^{\prime \prime}, 76-1,719 z$, daher die Länge von Rio Janeiro - $3^{\text {h }} 2^{\prime} 10^{\prime \prime}, 43+0,007 x$ $+1,719 z$. Die unbekannte Breitenverbesserung kann, da sie fast 0 beträgt, die Länge nicht bedeutend ändern; die Sonnenlänge ist durch eine Greenw. Beolbachtung verbessert worden, und $z$ drückt also nur noch die Verbesser. der Mondslänge aus.

Da der Unterschied der Längen zwischen Monte Video und hio Janeiro aus Beobachtungen bekannt ist, so fïge ich hier auch noch eine Beobachtung zur Bestimmung der Länge vou Monte Video hinzu. In den A.N. Nr.125. S.93 habe ich mehrere Beobachtungen des Durchganges des Mercurs durch die Somue von 1789 berechnet; ich trage hier noch die Bereclinung für Monte Video nach, die ich dort, als zu meinem Zweck nicht gehörig, weggelassen hatie.

Mercurdurcligang durch die Sonne 1789. 5 Nov. in Monte Video beohachtet.

Austr., innere Ber. $1^{\text {h }} 59^{\prime} 1^{\prime \prime}, 38 \mid 23^{\text {h }} 23^{\prime} 9^{\prime \prime}, 38+3,494 x$ Aus den Beobachtungen findet sich $x=-2^{\prime \prime}, 50$ und damit die verbess. Conj. für Montevideo $23^{\mathrm{h}} 23^{\prime} 0^{\prime \prime}, 65$. Hiernach eruibt sich, mit Anwendung der verbess. Conj., die Länge von Montevideo durch l'aris $-3^{\text {h }} 54^{\prime} 1^{\prime \prime}, 94$, Wien ....2 2",74, Ofen

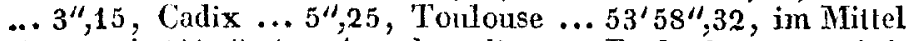
also $-3^{\text {h }} 54^{\prime} 2^{\prime \prime}, 24$. Aus eben diesen 5 Beobachlungen wird, nach Triesneckers Bercchnungen (Ephem. Vindob.1800 p.380) in der Conn. des tems pour 1830 p. 145 jene Länge im Mittel zu $-3^{\text {h }} 54^{\prime} 10^{\prime \prime}$, abgeleitet. Das Mittel aus meinen u. 'Thiesneckers Berechnungen ist $-3^{\text {h }} 54^{\prime} 6^{\prime \prime}, 2$. Nun fand Hr. Fonque chronometrisch den Längenunterschied zwischen Mlontevideo und der Insel Anhatomirim $7^{\circ} 34^{\prime} 18^{\prime \prime}, 3$ in Bogen ostwiirts, und zwischen der letztern Insel und der Insel Ratos (im Ifafen von Rio Janciro) $5^{\circ} 25^{\prime} 39^{\prime \prime}, 8$ östlich; demnach liegt die Insel liatos $12^{\circ} 59^{\prime} 58^{\prime \prime}, 1$ im Raum, oder $51^{\prime} 59^{\prime \prime}, 9$ in Zeit östlicher, als Montevideo. Der Capt. Heywood macht dagegen eben diesen Iängenunterschied $13^{\circ} 2^{\prime} 0^{\prime \prime}$, oder in Zeit $52^{\prime} 8^{\prime \prime}, 0$. Wird das Mitiel beider Bestimmungen, oder $52^{\prime} 4^{\prime \prime}, 0$, zur Länge von Montevideo $-3^{\text {h }} 54^{\prime} 6^{\prime \prime} 2$ addirt, so erhält man die Lünge der Inscl fiatos $=-3^{h} 2^{\prime} 2^{\prime \prime}, 2$. Eben diese Länge lälst sich aber auch durch den Längenunterschied mit Anhatomirim, nach Hrn. Fouque $=5^{\circ} 25^{\prime} 39^{\prime \prime}, 8$ bestimmen, da die Länge von Santa Catharina, Insel Anhatomirim, durch die wiederholten. und sorgfäligen Untersuchungen des Herrn Capt. Duperrey, Commandanten der Coquille, und des Ingenicur-Hydrographe, Hrn. Givry, welcher die Expedition des ITrn. Baron Roussin, Commandanten der Bayadère und des Favori, begleitele, mit einem hinreichenden Grade von Genauigkeit bekannt ist. Herr Civry setzt aus vielfachen von ilm selbst und den Officieren der
Jeiden Schiffe angestellten Beobachtungen die Länge von Anlıatomirim auf $-51^{\circ} 1^{\prime} 14^{\prime \prime}$ fest. (Conn. des tems 1825 und 1830). Herr Duperrey verainigt die Resultate von 892 Mondsdistanzen, während der Seefahrt der Bayadère beobachtet, nit 306 anderen, welche auf der Seefahrt der Coquille beobachtet wor'den; die ersten gaben $-51^{\circ} \mathbf{1}^{\prime} 13^{\prime \prime}, 6$, die audern $-51^{\circ} 0^{\prime} 6^{\prime \prime}, 2$ (Conn. d. t. 1830. p.182); das Millel, mit Rüclisicht auf die Anzahl der Beobachtungen, ist $-51^{\circ} 1^{\prime} 3^{\prime \prime}, 8$. Addirt man 21 dem Mittel aus beiden Bestimmungen der Herren Duperrey und Givry den östl. Längenunterschied $+5^{\circ} 25^{\prime} 39^{\prime \prime}, 8$, so erhïlt man die Länge der Insel Ratos $=-51^{\circ} 1^{\prime} 8^{\prime \prime}, 9+5^{\circ} 25^{\prime} 39^{\prime \prime}, 8$ $=-45^{\circ} 35^{\prime} 29^{\prime \prime}, 1$, oder in Zeit $-3^{\mathrm{h}} 2^{\prime} 21^{\prime \prime}, 9$.

Zum Schlusse stelle ich hier noch folgende Bestimmungen fïr die Länge von Rio Janeiro zur besseren Uebersicht zusammen:

1. Dic Länge von Montevideo als bekannt angenommen, gibt der Längenunterschied zwischen Montevideo und Anlictomirim, und dann zwischen dieser Insel und der Insel Ratos die Länge der Insel Ratos $=-3^{\text {h }} 2^{\prime} 2^{\prime \prime}, 2$.

2. Die Länge von Anhatomirim, als bekannt vorausgesetzt, folgt aus dem Längenunterschicd dieser Insel mit der Insel Ratos die Länge der letztern $=-3^{\mathrm{h}} 2^{\prime} 21^{\prime \prime}, 9$.

3. Herr Givry setzt die Länge der Insel Ratos, als Resullat sehr zahlreicher, von ihm selbst und mehreren Secofficicren an der Brasilianischen Küste nord - und sïdwärts von Rio Janeiro genommener Mondsdist. $=-45^{\circ} 35^{\prime} 14^{\prime \prime}$ oder in Zeit - $3^{\text {h }} 2^{\prime} 20^{\prime \prime}, 9$.

4. Mondsdistanzen von den Herren Rümker und Brisbane in Butofago beubachtet, geben dessen Länge $-3^{\mathrm{h}} 2^{\prime} 3^{\prime \prime}: 1$ und da, nach chronometrischen Beobachtungen die Insel Ralos $3^{\prime \prime}$ in Zeit östlicher liegt, Länge dieser Insel $-3^{\text {h }} 2^{\prime} 0^{\prime \prime}, 1$ (Astr. Nachr. Nr.5).

5. Die oben berechncte Sonnenfinsternifs vom 20. Febr. 1784, durch Dorva beobachtet *), gilst Länge des Schlosses zu hio Janeiro $-3^{\text {h }} 2^{\prime} 10^{\prime \prime}, 4$.

6. Dorta selbst gibt als Resultat seiner verschiedenen Beobachtungen die Läıge des Schlosses zu Rio Janeiro in den Memorias da Acad. Feal das Sciencias de Lishoa, 'Tom. I. $=-3^{\mathrm{h}} 2^{\prime} 31^{\prime \prime}, 3$. (s. Monatl. Corresp. IV.B. S. 352)

Die von Brisbane beobachtele Bedeckung des Doppelsterng (s. oben) glaubte ich hier übergehen zu müssen, da sie cine gar zu sehr abwcichende Iä̈nge gibt. Das Mittel aus den Bestimmungen Nr. $1-3$ gibt $-3^{\mathrm{h}} 2^{\prime} 15^{\prime \prime}, 0$ für die Länge der Insel Ratos, und das Mittel aus N. 1-6 ohne Unterschied genommen, gibt $3^{\text {h }} 2^{\prime} 14^{\prime \prime}, 5$. Dic Länge der Insel Ralos kann also nach den bisher bekannt gewordenen Bestimmungen mit einiger Wahrscheinlichkeit $=-3^{\natural} 2^{\prime} 15^{\prime \prime}$ gesetzt werden.

*) Ich bemerke noch, dafs Dorfa diese Finsternifs doch nicht so gänzlich nahe am Horizonte beobachtet haben murs. Die Sonne gieng am 20 Febr. 1784, mit Rücksicht auf Refraction und Sonnenhalbmesser, in Rio Janeiro unter nm 6h 22' wahre Zeit; der Anfang wurde von Dorta um 5h 51' 14" w. Z. benbachtet, demnach eine halbe Stunde vor Sonnentuntergang, als die Sonne noch $6^{\circ} 11^{\prime}$ hoch stand.

Stutgart, den 15 May 1828.

$$
\text { W } u r m
$$

